\title{
Integration of E-learning into the Physiology Education of Medical Students in their Pre-clinical Curriculum
}

\author{
Radha Patel ${ }^{a}$ Kelly J Kovacs ${ }^{a}$ Christopher Prevette ${ }^{a}$ Tian Chen ${ }^{b}$ Coral D Matus ${ }^{a}$ and Bindu Menon (D) $a$
}

Coresponding author(s): Bindu.Menon@utoledo.edu

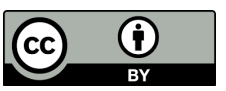

\footnotetext{
${ }^{a}$ Department of Medical Education, University of Toledo College of Medicine and Life Sciences, Toledo, Ohio 43614,, and ${ }^{b}$ Department of Mathematics and Statistics, University of Toledo, Toledo $\mathrm{OH} 43614$
}

\begin{abstract}
Purpose: In our institution, we initiated integrated learning in the format of Learning Modules (LM), interactive audio/visual modules developed by the faculty in alignment with session learning objectives, as pre-work for in-class sessions. This pilot study examined students' perceptions of this new learning method and effectiveness in helping them achieve content mastery. Methods: The instructor provided the LMs in advance, allowing a selfpaced introduction of critical concepts that were subsequently discussed in detail during the in-class learning (ICL) session. A cognitive diagnostic assessment was used to analyze the student's performance on the exams. This involved identifying six skills, one or more of which were marked to be necessary for answering each exam question correctly. A question-by-skill Qmatrix was constructed, followed by analysis using a Deterministic Input, Noisy "And" Gate (DINA) model. Results: $70 \%$ of the students rated the new approach as "Excellent or Good." $63 \%$ of the students attained skills necessary to answer questions that involved integrating information gained separately from LM and ICL sessions. However, only $28 \%$ of the students achieved mastery in all the six skills. Conclusion: This integrative learning system allowed for time optimization since ICL sessions could focus on more interactive aspects of the content.
\end{abstract}

self-learning | medical physiology | undergraduate medical education | learning modules

T he implementation of active self-directed learning (SDL) has become an integral part of academic learning at all levels of education. Active SDL is particularly gaining interest and becoming a salient feature in many medical schools throughout the United States due to the emphasis on SDL for Liaison Committee on Medical Education (LCME) accreditation. Additionally, when following the Cognitive Flexibility Theory of knowledge acquisition principles, there is a significant positive contribution to the learner's mas- tery of content $(1,2,3)$.

The concept of active SDL has been thought of as an "approach where learners are motivated to assume personal responsibility and collaborative control of the cognitive and contextual processes in constructing and confirming meaningful and worthwhile learning outcomes" (4). This form of directed learning requires students to participate in structured activities designed by teachers while individually engaging with the material (5). Active SDL has given students the ability to review material at their own pace and allows educators to cover more material through in-person and online learning. Through this approach, students engage with the material on an individual level, encouraging them to go beyond mere memorization and further into comprehension and application of the material (5).

E-learning is a form of active SDL that involves the "delivery of education through Information and Communication Technology (ITC)" $(5,6)$. The employment of E-learning in medical education has been suggested to positively affect learning outcomes and allow students agency over their learning (7). The current study examined the use of E-learning in the format of Learning Modules (LM) [defined internally as interactive audio/visual modules developed by faculty in alignment with course learning objectives] to introduce focused SDL for the medical students in their second year. We examined the assessment outcomes to study the effectiveness of the new method. This study shows that E-learning is a learning enhancer, when used as a facilitating feature integrated into the traditional medical education model.

Submitted: 06/01/2021, published: 07/02/2021. 


\section{Materials and Methods}

Approach: The new integrated method was introduced to the second-year (M2) class of medical students in their foundational science curriculum (class size, 177) during the gastrointestinal (GI) physiology sessions. The course instructor assigned short e-learning modules (LMs) to the students in preparation for traditional lecture style in-class sessions.

Preparation of LMs: The LMs were prepared on the Articulate 360 Rise TM platform. Each LM is expected to take approximately 15-30 minutes for completion. The LMs also had short quizzes incorporated into them to assess knowledge acquisition. It was clearly communicated to the students that the LMs were required pre-work to be completed before the in-class session. Since the LMs were delivered through the online learning management system, Blackboard TM, the instructor could access important analytical data such as the number of times each student viewed the LMs and the time spent on each view.

Survey: The researchers conducted a short closed-ended onequestion survey to assess student satisfaction with the integrated pedagogies. The survey was administered via the online Poll Everywhere TM software and was open to everyone that attended the in-class session. The overall response rate was $31 \%$. All submissions were reported anonymously.

Assessment Outcome: We used a cognitive diagnostic assessment to analyze students' performance on the final course exam. The instructor identified six skills, one or more of which were marked to be necessary for answering each exam question correctly (Table 1). A question-by-skill Q-matrix was constructed. For example, we determined that to answer a question on gastroesophageal reflux disease, students must remember the sphincter muscle's role at the lower end of the esophagus and needed to possess two skills: S1 and S3 (see Table 1 for skills). Then, a Deterministic Input, Noisy "And" Gate (DINA) model were used to analyze the data (8) of the entire student body $(\mathrm{n}=177)$ and estimate the percentage mastery for each skill. The student performance data from 12 questions on this content that appeared in the final course exam were analyzed similarly using this method.

Table 1. The various skills which the students must possess to correctly answer the assessment items from GI physiology that appeared on the course exam.

\begin{tabular}{|c|c|}
\hline Student & Skills \\
\hline S1 & Recall the knowledge of the normal function of the GI tract gained from the in-class sessions (ICL) \\
\hline S2 & Recall the knowledge gained from e-learning modules (LM) on the basic structure and function of the GI tract \\
\hline S3 & Ability to apply the knowledge of GI physiology learned from the ICL to solve a clinical problem \\
\hline S4 & Ability to apply the knowledge of GI physiology learned from the LMs to solve a clinical problem \\
\hline S5 & Ability to integrate the knowledge gained separately from the ICLs and LMs and develop a broad understanding of GI physiology \\
\hline S6 & Ability to integrate the knowledge of physiology concepts gained separately from the ICLs and LMs to solve a clinical problem \\
\hline
\end{tabular}

\section{Results}

The survey results showed that the majority of the students appreciated the new method; $25 \%$ of the responders gave an "excellent" (4 out 4) rating, while 55\% thought it was "Good" (rating of 3 out 4 ). Only $5 \%$ thought it was below average (1 out of 4$)$. The results of the DINA model analysis are shown in Figure 1. We found that $28 \%$ of students acquired complete mastery in GI Physiology content; they attained mastery in all six skills. Mastery in skill 5, which required integration of knowledge from the two separate pedagogies, was acquired by $63 \%$ of the students.

Similarly, skill 6 , which required the application of information obtained from the entire course to solve a real-life problem in a clinical scenario, was mastered by $64 \%$. A significant number of students who mastered skills 5 and 6 had also attained mastery in the ICL session content, thus stressing the importance of in-class sessions. It is important to note that none of the students who did not use institutional resources, by not utilizing the LMs and not attending the in-class sessions (49\%), attained complete mastery.

\section{Discussion}

As our technology advances, so do the methods by which we can process and disseminate information; learning now is not limited to geographic location and has given students more autonomous access to information. With this increased agency over ones' learning comes the need for adaptive learning styles that encourage students to become life-long active, self-directed learners. Through the advancement of learning aids, students can acquire and master critical skills beyond in-class learning. SDL is a central concept 
in adult learning and practice that integrates self-management, selfmonitoring, and motivation (4). Our medical school's integrated curriculum accounts for SDL throughout a students' progression from the preclinical to clinical years. The application of the LMs is in response to the changes in the LCME standard, redefined as "The faculty of a medical school ensure that the medical curriculum includes self-directed learning experiences and unscheduled time to allow medical students to develop the skills of lifelong learning" (Reference: Functions and Structure of a Medical School; Standards for Accreditation of Medical Education Programs Leading to the M.D. degree).

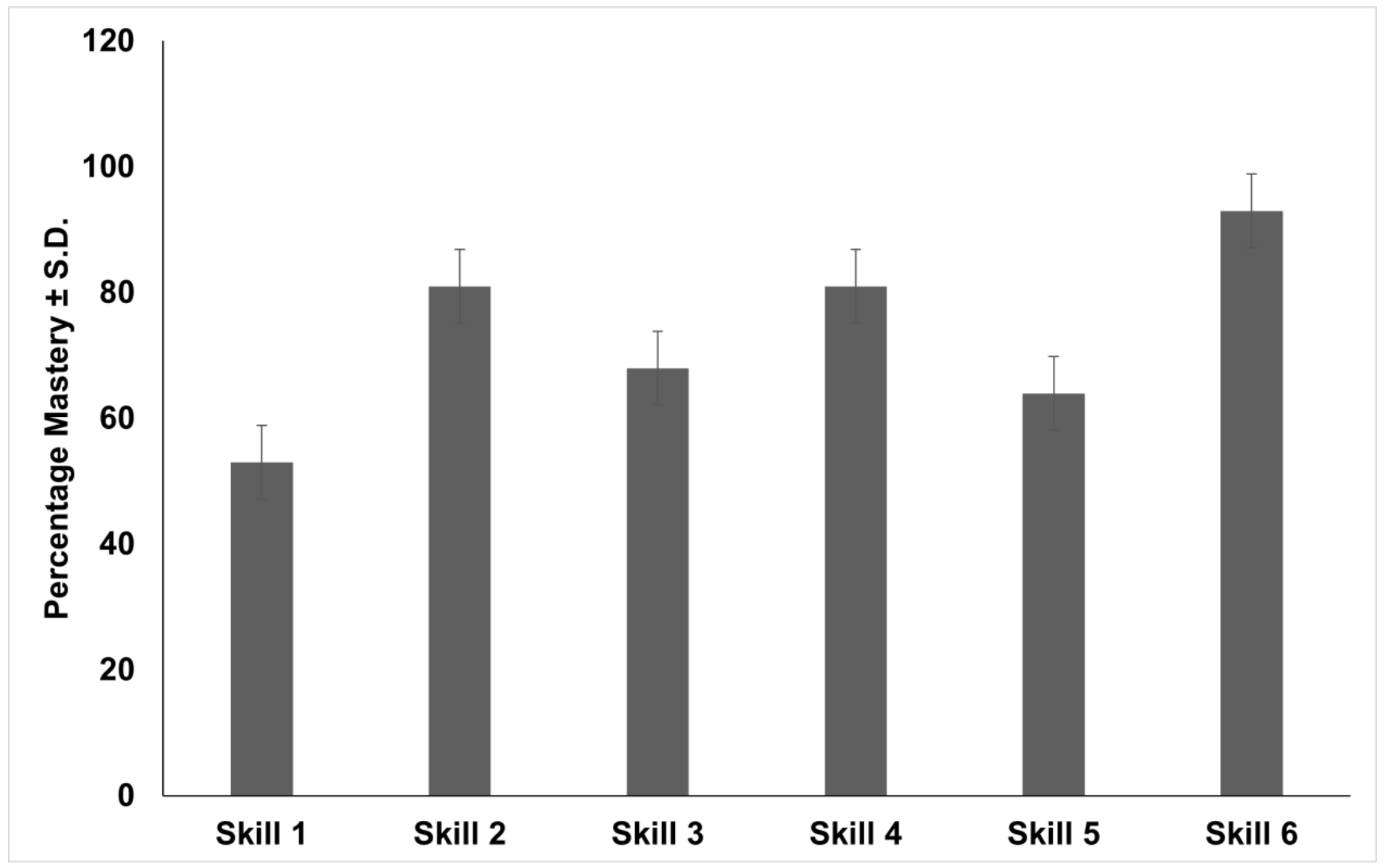

Fig. 1. The cognitive diagnostic assessment. The X-axis shows the various skills necessary to answer the multiple questions on the GI physiology exam successfully. Y-axis gives the percentage of students who achieved mastery in the corresponding skills.

SDL involves medical students' self-assessment of learning needs; independent identification, analysis, and synthesis of relevant information; appraisal of the credibility of information sources; and feedback on these skills (Reference: Functions and Structure of a Medical School; Standards for Accreditation of Medical Education Programs Leading to the M.D. degree). SDL is based on the principle that learner exercises independence in deciding what is worthwhile to learn and how to approach the task to reach the desired outcomes (4). It is defined as an approach where learners are motivated to assume personal responsibility and collaborative control of the cognitive (self-monitoring) and contextual (selfmanagement) processes in constructing and confirming meaningful and worthwhile learning outcomes. However, it is also believed that there is no standard way of implementing a curriculum focused around self-directed learning, but experts have proposed a model that integrates the metacognitive theory of learning $(9,10)$. In this approach, the learners are to be provided with choices of how they wish to carry out the learning process proactively; "material resources should be available, approaches suggested, flexible pacing accommodated, and questioning and feedback provided when needed (11). We incorporate this into our model by allowing students to exercise focused self-directed learning in preparation for the in-class sessions. In other words, we complement independent student learning by providing credible material in the form of LMs to aid in the synthesis of information relevant to their identi- 
fied needs. While completion of LM's is not tracked or awarded a grade, we strongly recommend them as a learning source. We do not discourage students from using other resources instead of LMs. However, the central framework on which the SDL should be focused is given in the form of learning objectives disseminated at the beginning of the LMs.

Our intended outcomes were to assess the efficacy of learning modules and in-class learning on mastery of core concepts. The assessments covered core concepts of the reformed systems-based curriculum at the University. A total of six binary skills were assessed when answering the exam questions from the GI physiology curriculum. These skills consisted of recollection of information, applying information, synthesizing information, and integrating information from the in-class learning and e-learning modules. They were categorized to assess a student's ability to build upon each essential skill and ultimately incorporate information from both learning materials to achieve mastery of the content. Our results show that better acquisition of content mastery was found to stem from elearning modules ( $81 \%$ mastery in skill 2; fig 1) instead of in-class learning sessions (53\% mastery in skill 1). However, we caution that faculty guidance is needed to integrate the information from the learning modules with in-class learning for complete mastery of the clinical application of knowledge.

However, it is of concern that only $28 \%$ of the students achieved complete mastery. This could be attributed to the sessions not being made as mandatory. Several of the students chose not to use the institutional resources (49\%). It would be interesting if we could analyze similar data from the NBME formatted end-of-course exams and see if there is a correlation to the observed trend in that exam. More importantly, one of the commonly acknowledged shortcomings of the pass-fail system is that students can still pass the exam even if they do not acquire complete mastery of the skills.

\section{Limitations and Next Steps}

This pilot study was conducted as a first step in the introduction of SDL in our institution. Two critical components of SDL, as defined by the LCME, are missing from our model; \share the information with their peers and supervisors" and \receive feedback on their information-seeking skills". Currently, we are in the process of introducing a platform for the students to share with their peers the knowledge acquired from various sources such as LMs. The other

1. Spiro, RJ, RL Coulson, PJ Feltovich, and D Anderson 1988 Cognitive flexibility theory: Advanced knowledge acquisition in ill-structured domains. Hillsdale, NJ: Erlbaum.

2. Spiro, RJ, PJ Feltovich, MJ Jacobson, and RLIE Coulson 1992 Cognitive flexibility, constructivism and hypertext: Random access instruction for advanced knowledge acquisition in ill-structured domains. Hillsdale, $\mathrm{NJ}$ : Erlbaum.

3. Spiro, RJ, and Jle Jehng 1990 Cognitive flexibility and hypertext: Theory and technology for the non-linear and multidimensional traversal of complex subject matter. Hillsdale, NJ: Erlbaum.

4. Garrison, DR 1997 Self-Directed Learning: Toward A Comprehensive Model. Adult Education Quarterly 48 18-33.

5. Gleason, BL, MJ Peeters, BH Resman-Targoff, S Karr, S McBane, K Kelley, T Thomas, and TH Denetclaw 2011 An active-learning strategies primer for achieving ability-based educational outcomes. Am J Pharm Educ 75186.

6. Lawn, S, X Zhi, and A Morello 2017 An integrative review of e-learning in the delivery of self-management support training for health professionals. BMC Med Educ 17183. missing element, providing feedback to the students, will also be introduced in the next step of the implementation.

Another limitation of the study is that it is a preliminary study and uses data from students in the 2018-2019 academic year. We are currently in the process of analyzing data from the students in the more recent classes (2019-2020 and 2020-2021) as well as previous years to examine the new method's effectiveness in acquiring and retrieving knowledge. Once we have obtained more comprehensive data, it will provide more critical information about the utility of LMs in student learning.

\section{Conclusion}

This pilot study has two major components that contribute significantly to the field of medical education. We show that providing source material for SDL in the form of LMs can be an efficient learning method to acquire and retain information considering the challenges posed by the ever-expanding wealth of knowledge. Secondly, quantitative analyses, such as the kind used in this study, can actively assess curriculum strengths and weaknesses based on students' performances and content mastery. Thus, it allows instructors to refine their teaching approaches and deliver more effective and streamlined teaching material, giving them a more focused clinical knowledge and mastery approach.

\section{Conflict of interest}

Authors declare no conflict of interest.

\section{Institutional Review Board approval}

All analysis and survey involving the students were in accordance with the standards of the institutional research committee (University of Toledo Institutional Review Board). The study was granted exempt status by the IRB.

\section{Authors' contributions}

Conceptualization: C.M, and B.M. Methodology: C.P and K.J.K. Data curation: K.J.K; Analysis: T.C and B.M; Writing: original draft: R.P; review and editing: K.J.K and B.M.

7. Fransen, F, H Martens, I Nagtzaam, and S Heeneman 2018 Use of e-learning in clinical clerkships: effects on acquisition of dermatological knowledge and learning processes. Int $J$ Med Educ 9 11-17.

8. Bangeranye, C, and YS Lim 2020 How to Use Cognitively Diagnostic Assessments of Student Performance as a Method for Monitoring and Managing the Instructional Quality in Undergraduate Medical Education. Acad Med 95 145150.

9. Flavell, JH 1976 Metacognitive aspects of problem solving. Hillsdale, NJ: Erlbaum.

10. Flavell, JH 1979 Metacognition and cognitive monitoring: A new area of cognitive-developmental inquiry. American Psychologist, 34906 - 911.

11. Hurford, D, and A Read 2011 Do podcasts and screencasts enable or hinder independent learning. Practitioner Research in Higher Education 5 30-38. 